\title{
Formal Pericyclic-Coupled Electron Transfer: I. Stepwise Formal Diels-Alder Cycloaddition Enabled by Addition-Coupled Electron Transfer
}

Yumiao $\mathrm{Ma}^{* \mathrm{a}, \mathrm{b}}$

a. BSJ Institute, Haidian, Beijing, 100084, People’s Republic of China. ymma@bsj-institute.top

b. Hangzhou Yanqu Information Technology Co., Ltd. Xihu District, Hangzhou City, Zhejiang Province, 310003, People’s Republic of China

\begin{abstract}
The formal Diels-Alder (D-A) reaction of alkenes with 1,2-diimine and related substrates is designed and studied by density functional theory (DFT), multi-reference and quasi-classical trajectory calculations in the presence of a benzoquinone-based organic oxidant. The reaction is found to occur through an Addition-Coupled Electron Transfer (ACET) with one electron transferred to the oxidant in one elementary step, affording a carbon-radical, followed by an intramolecular radical addition. The overall barrier is significantly reduced as compared to the traditional concerted D-A reaction. No concerted Pericyclic-Coupled Electron Transfer (cPeriCET), the reaction with two new bond formation events in one elementary step coupled with electron transfer, was found.
\end{abstract}

\section{Introduction}

Pericyclic reactions ${ }^{1}$, and of special interest in this work, Diels-Alder cycloaddition reactions, have been a well-known family of fundamentally important reactions with broad mechanistic variety, both concerted and stepwise, synchronous and asynchronous, with and without entropy intermediates ${ }^{2-10}$. In most cases, the reaction involving unactivated olefins are challenging due to its high barrier. The reactivity is known to be promoted in the presence of redox active environment, known as oxidative cycloaddition ${ }^{11-15}$ and reductive cycloaddition ${ }^{16-19}$. Most of these reactions make use of a redox-active metal, and are made up by multiple elementary reactions with great mechanistic diversity. Despite these advances, it is still a question whether a single electron transfer event can be coupled in the same elementary step of a concerted or a formal cycloaddition.

In our previous report, we have found that nucleophilic addition can be coupled to a single electron transfer in one elementary step, resulting in an Addition-Coupled Electron Transfer (ACET) ${ }^{20}$. In this work, we aim to answer the question whether a similar coupling could occur in the step of a cycloaddition reaction. By designing a cycloaddition reaction between $\mathbf{1}$ and ethylene, and other related examples, we found that the reaction follows a stepwise ACET and radical cyclization mode, rather than traditional concerted cycloaddition or an imaginary "concerted Pericyclic-Coupled Electron Transfer" (cPeriCET). 
(a)

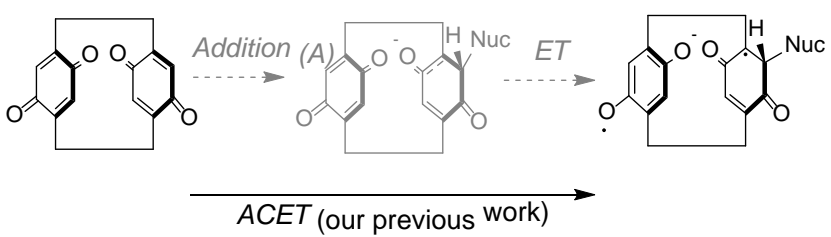

(b)<smiles>N#CC1=C(C#N)C(=O)C(CC(=[W])C=[W])=CC1=O</smiles>

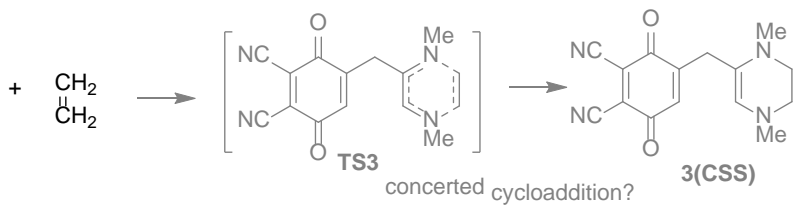

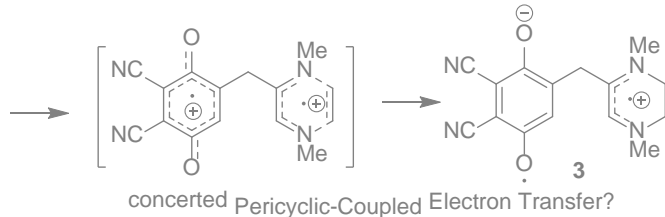

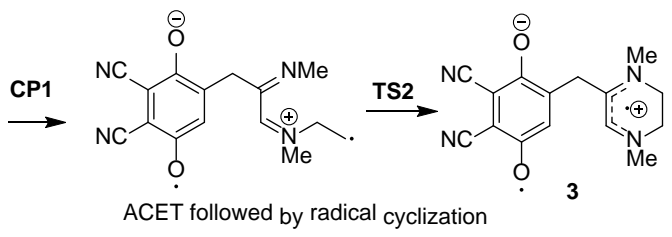

Figure 1. A summary of this work. $\mathrm{TS}=$ transition state, and CP $=$ crossing point. CSS $=$ close-shell singlet state.

\section{Results and Discussions}

The model substrate $\mathbf{1}$ is designed by the following considerations: 1 . A p-benzoquinone motif with strong electron-withdrawing groups could act as an intramolecular oxidant, and the intramolecular nature avoids troubles with the flexible binding conformation related to intermolecular oxidant. The oxidant is linked to the center of the cycloaddition reaction by a $\mathrm{CH}_{2}$ linkage, preventing direct influence on the electronic properties of the reaction center. 2 . The 1,2-diimine unit is taken as the diene partner due to its inertness toward oxidation. However, once the cycloaddition occurs, it will be transformed into a strongly reductive 1,2-diaminoethylene product 3 , and is expected to be prone to the electron transfer to the benzoquinone unit.

The Gibbs free energy profile for the cycloaddition of $\mathbf{1}$ to ethylene exhibits the co-existence of traditional concerted cycloaddition pathway and ACET-involved pathway (Figure 2). The substrate and ethylene form a pre-activation complex 1Comp in its close-shelled singlet state (CSS), with a slight endothermicity of $4.0 \mathrm{kcal} / \mathrm{mol}$. The corresponding triplet state is quite high in energy (52.9 $\mathrm{kcal} / \mathrm{mol}$ ). The high energy of the triplet or open-shelled single (OSS) state of 1Comp is further validated by N-Electron Valence State Perturbation Theory (NEVPT2) calculation ${ }^{21}$, which gives the relative triplet and OSS energy at 53.3 and $49.8 \mathrm{kcal} / \mathrm{mol}$, respectively. The excellent quantitative consistence between NEVPT2 and DFT calculations supports the reliability of DFT on this system. The concerted cycloaddition through TS3 on the CSS potential energy surface (PES) requires a high barrier of $40.7 \mathrm{kcal} / \mathrm{mol}$, affording the product ${ }^{1} \mathbf{3}$ (CSS) with the CSS state as a stable wave-function. Although there is a crossing point from CSS-PES to triplet-PES near TS3, it is higher in energy by $4.8 \mathrm{kcal} / \mathrm{mol}$, rendering it unimportant. 
At the meantime, there are two other pathways involving open-shelled species. Once ${ }^{\mathbf{1}} \mathbf{1 C o m p}$ enters the PES of the open-shelled singlet (OSS) or triplet state through the crossing point from the CSSPES, namely CP1(CSS_OSS) and CP1(CSS_Triplet), respectively, a biradical intermediate $\mathbf{2}$ will form, in which only one $\mathrm{N}-\mathrm{C}$ bond (namely $\mathrm{N} 1-\mathrm{C}$ ) forms. The moderate spin-orbit coupling matrix element between CSS and triplet state at $\mathbf{C P 1}$ (CSS_Triplet) of $3.18 \mathrm{~cm}^{-1}$ indicates that the change of spin state is possible. Notably, the transition state (TS) on the single OSS or triplet-PES was unable to be located, and the reaction is suggested to occur directly through CP1 instead of a TS, which will be further discussed later. While $\mathbf{2}$ is not a minimum on the CSS-PES, the minimums of OSS and triplet state of $\mathbf{2}$ are nearly degenerated, and this feature is shared by TS2 and $\mathbf{3}$. The second $\mathrm{N}-\mathrm{C}$ bond formation occurs through a radical addition of the carbon-centered radical to N2, with a very low barrier of $\sim 3.0 \mathrm{kcal} / \mathrm{mol}$ for both the OSS and triplet state. The resulting product ${ }^{3} \mathbf{3}$ and ${ }^{1} \mathbf{3}$ (OSS) are much lower in energy than its CSS analogue $\left(-41.6 \mathrm{kcal} / \mathrm{mol}\right.$ for ${ }^{3} \mathbf{3}$ versus -17.5 $\mathrm{kcal} / \mathrm{mol}$ for ${ }^{1} \mathbf{3}(\mathrm{CSS})$ ), and shares a planar 1,2-diaminoethylene unit, on the contrary to the nonplanar structure of ${ }^{\mathbf{1}} \mathbf{3}$ (CSS). This change in geometry is in consistence with an oxidized 1,2diaminoethylene unit.

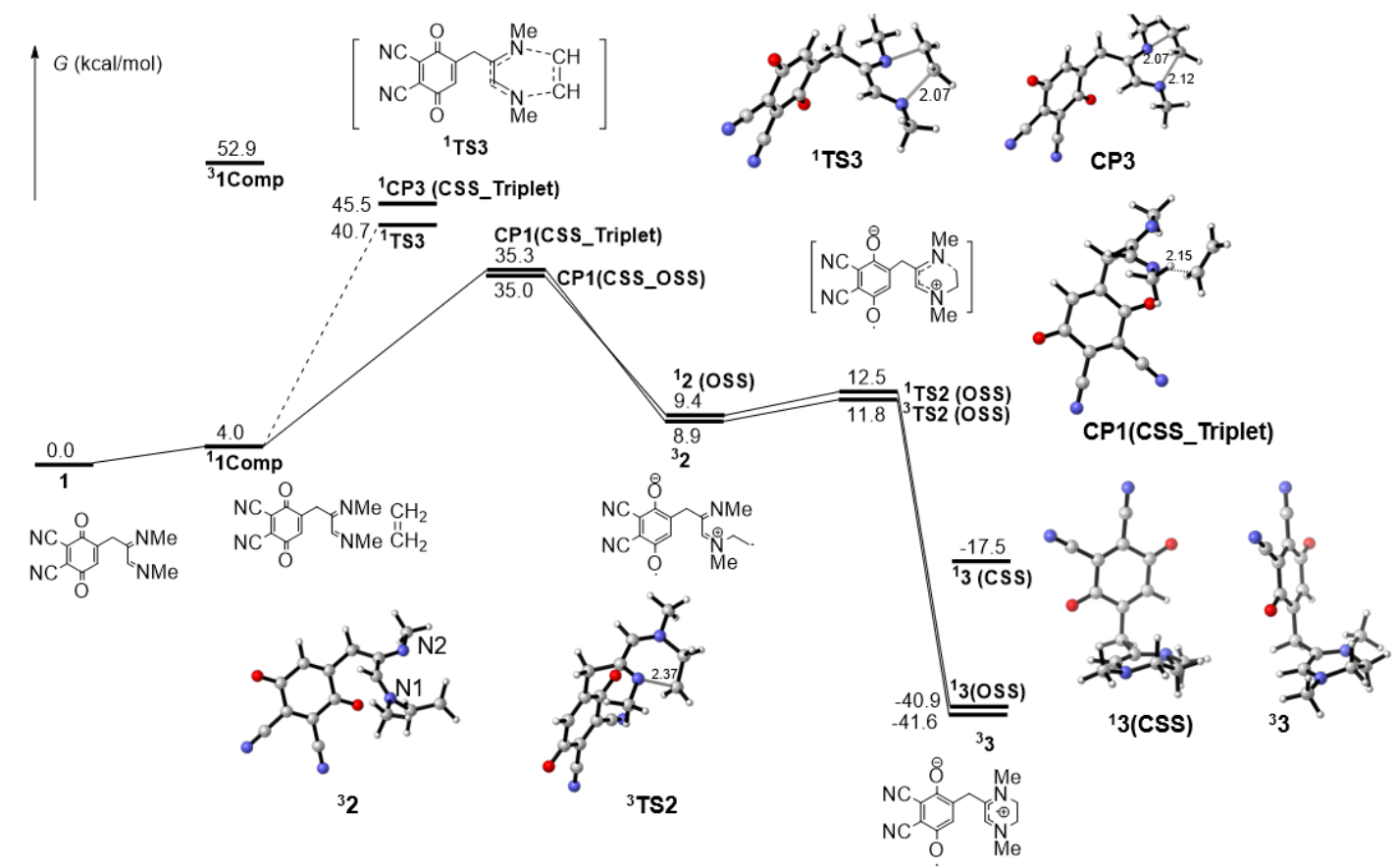

Figure 2. The Gibbs free energy profile for the formal cycloaddition reaction between $\mathbf{1}$ and ethylene. Distances are in angstrom.

The key point to distinguish ACET from ET-A or A-ET in the first N-C bond formation step is the existence of related intermediates. Since the addition product $\mathbf{2}$ is not a minimum on the CSS-PES, the first $\mathrm{N}-\mathrm{C}$ bond formation must not be a nucleophilic addition followed by electron transfer (AET). Then the next question is whether the crossing point from CSS-PES to open-shelled PES, namely CP1, is able to lead the substrate to 2 . If so, the addition will happen once the crossing into open-shelled PES occurs, and the stepwise ET giving high-energy intermediate ${ }^{3} \mathbf{1 C o m p}$ will be avoided. Starting from both CP1(CSS_OSS) and CP1(CSS_Triplet), the downhill pathway was generated on both the two PESs (Figure 3a for CP1(CSS_Triplet) and Figure S1 for 
CP2(CSS_OSS)). A downhill pathway is produced in a similar method to Intrinsic Reaction Coordinate (IRC), and is known to reflect the minimum energy reaction path crossing the starting point. With CP1(CSS_Triplet) as an example, it is clear that this species lies on the reaction path towards ${ }^{1} \mathbf{1 C o m p}$ and ${ }^{3} \mathbf{2}$ on the CSS and triplet-PES, respectively. The similar situation is shared for CP1(CSS_OSS). Therefore, it is concluded that CP1 connects indeed ${ }^{\mathbf{1}} \mathbf{1 C o m p}$ and $\mathbf{2}$. As a result, there is no need for a first separated ET to give ${ }^{3}$ 1Comp; $\mathrm{N}-\mathrm{C} 1$ formation coupled by electron transfer occurs through a crossing point rather than a TS, and is therefore an ACET reaction.

The electronic structure for the key species is studied by both the spin density distribution and multireference methods. The OSS and triplet species are quite similar, not only with respect to its energetics as seen in Figure 2, but also in terms of spin density distribution and geometry. With CP1 as an example, when the geometry of CP1(CSS_OSS) and CP1(CSS_Triplet) is placed together, it can be seen that they overlap well, indicating the quite similar geometry (Figure 3b). According to the spin density isosurface, it is quite clear that $\mathbf{2}$ is a biradical at both C2 and the benzoquinone unit, and the spin density is further distributed over the 1,2-diaminoethylene part in $\mathbf{3}$. The spin density of CP1(CSS_Triplet) on its triplet state is located on both N1 and C2. The results from DFT calculations were further validated by NEVPT2 calculation with an active space size at 10 electrons and 10 orbitals. Based on the DFT-located crossing point geometry, the OSS, CSS, triplet states are quite close to be degenerated on the NEVPT2 level, supporting the correctness of DFT-located crossing points. The electronic configurations of both the OSS and triplet state are dominated (contributed by $>99 \%$ ) by the HOMO-LUMO excitation. According to the Completed Active Space Self-Consistent Field (CASSCF) optimized orbital, it is clear the HOMO and LUMO is contributed by the benzoquinone unit and the $\pi$ orbital of ethylene slightly mixed with N1 lone pair, respectively. These results are clearly in consistence with the single electron transfer into the benzoquinone. 


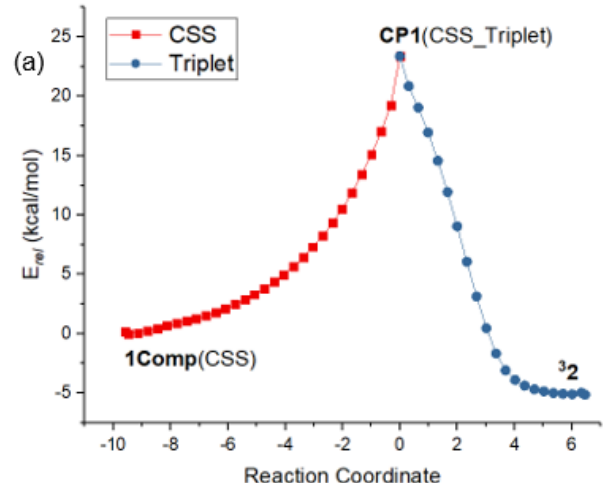

(c) (b)

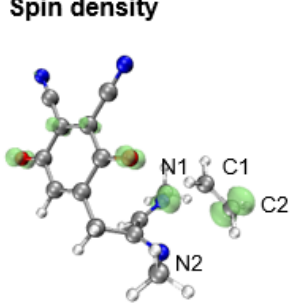

CP1(CSS_Triplet)

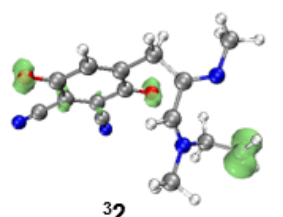

32

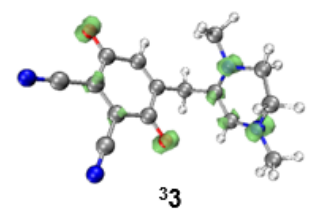

33

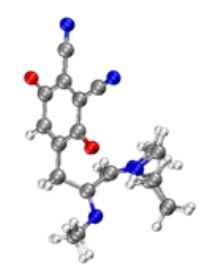

Superposed geometry of the two CP1s
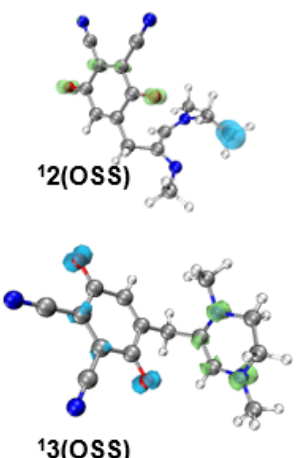

13(OSS)

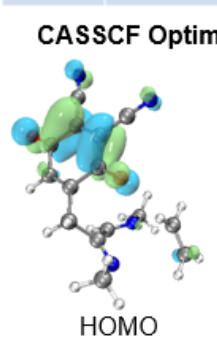

\section{tals}

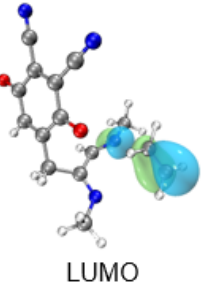

Figure 3. (a) The downhill paths starting from CP1(CSS_Triplet) on the CSS and triplet-PES. (b) The spin density isosurfaces (isovalue of 0.02 a.u.) for selected species. (c) The NEVPT2 relative energies for the interested states of CP1s, with the triplet state of CP1(CSS_Triplet) as the zero point. The isosurface for the CASSCF-optimized natural orbital is shown at the bottom.

In order to further study the behavior of the system upon going through CP1, quasi-classical molecular dynamics trajectories were generated starting from CP1(CSS_Triplet). All the trajectories were initiated according to the vibrational modes on the CSS-PES. After initiation, for each of the points along a trajectory, CSS, OSS and triplet states were calculated, and the one with lowest energy was taken for the propagation of the next point. 26 trajectories were generated in total, of which 8 trajectories led to the formation of $\mathbf{2}$ with an averaged timing of $30 \mathrm{fs}$, and the rest gave dissociated substrates (averaged timing of $41 \mathrm{fs}$ ). The intermediate 2 in all of the 8 productive trajectories remains stable within the time period of $200 \mathrm{fs}$, indicating that no dynamical concertedness between the two $\mathrm{N}-\mathrm{C}$ bond formation events occurs in this time scale. A typical trajectory was selected for showing the evolution of the energetics of OSS, CSS and triplet state in Figure 4. For a typical productive trajectory, the triplet and OSS states remain near-degenerated along the whole trajectory. Although they are also near-degenerated with CSS near the initiation of the trajectory, the open-shelled states gain stability rapidly (in $10 \mathrm{fs}$ ), and no re-crossing into the CSS state is observed. 
(a)

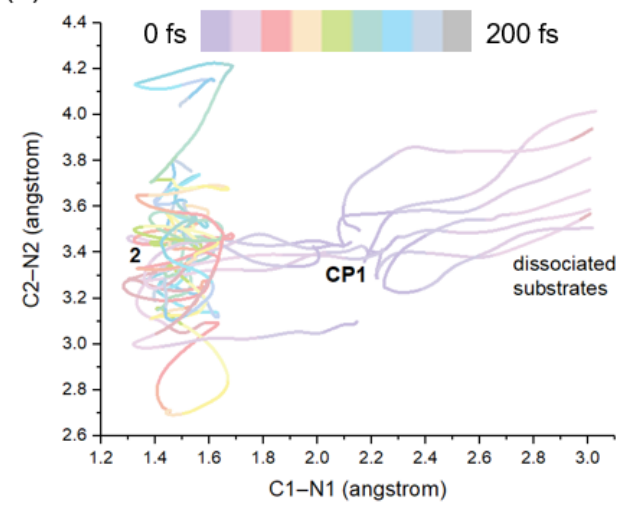

(b)

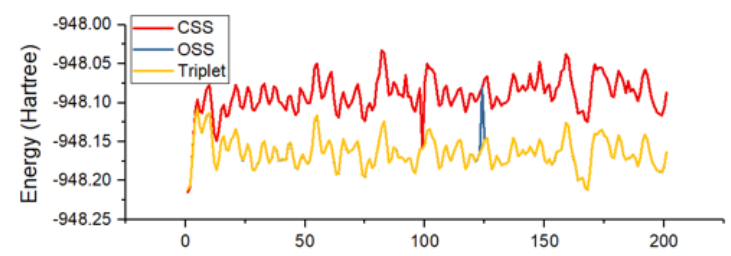

Figure 4. The evolution of (a) interested bond lengths and (b) energetics of interested states along selected trajectories. The line for OSS almost overlaps with the triplet state.

According to the ACET-involved mechanism established above, the reaction barrier can be considered to be the relative free energy of CP1 with $\mathbf{1}$ as the zero point, although the first addition does not occur through a transition state. It is naturally proposed that the position of $\mathbf{C P 1}$ along the reaction coordinate, as well as its energetics, is influenced by both the oxidizing ability of the benzoquinone motif and the electron-richness of the cycloaddition center. The relative Gibbs energy of CP1(CSS_Triplet) was thus examined for selected alkene (Figure 5). In general, it is seen that for an electron-rich alkene the energy of CP1 is lowered, although efforts to establish simple quantitative relationship between CP1 energetics and the HOMO level or redox potential of alkenes failed. Notably, for the very electron-deficient 1,1-dicyanoethylene, the mechanism for the first C$\mathrm{N}$ bond formation has been shifted from ACET into A-ET process (see Figure S2 for details), because of its strongly stabilized close-shelled addition product by the electron-withdrawing cyano groups. Besides, Lewis acids coordination into the benzoquinone unit strongly stabilizes CP1, leading to a much lower $\mathbf{C P 1}$ energy of $21.1 \mathrm{kcal} / \mathrm{mol}$ for $\mathrm{AlCl}_{3}$ (with the o-benzoquinone derivative $\mathbf{4}$ as the substrate) and $24.3 \mathrm{kcal} / \mathrm{mol}$ for $\mathrm{BF}_{3}$ (with $\mathbf{1}$ as the substrate). The substrate $\mathbf{4}$ was originally designed to explore the possibility of two-electron-transfer in the formal cycloaddition reaction: Lewis-acid coordinated o-benzoquinone might act as two-electron oxidant to be transformed into catecholate dianion. However, no two-electron coupled cycloaddition was found, and the reaction was determined to be again an ACET coupled with a single electron transfer. 

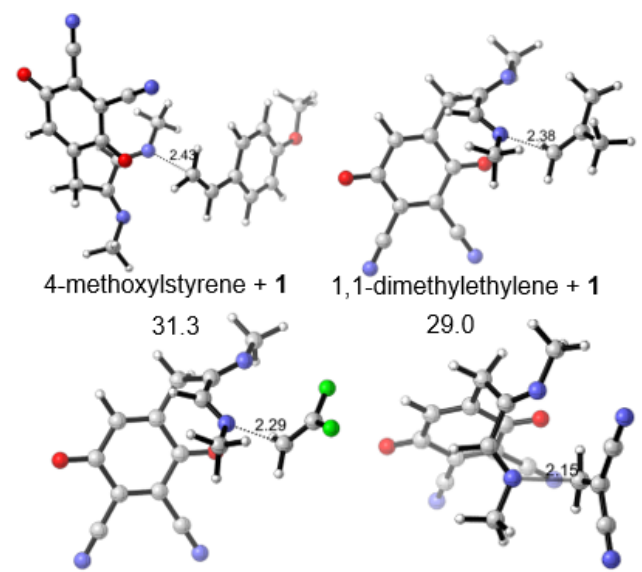

1,1-difluoroethylene + 1

1,1-dicyanoethylene +1 39.6 11.0 (TS for first addition, CSS)
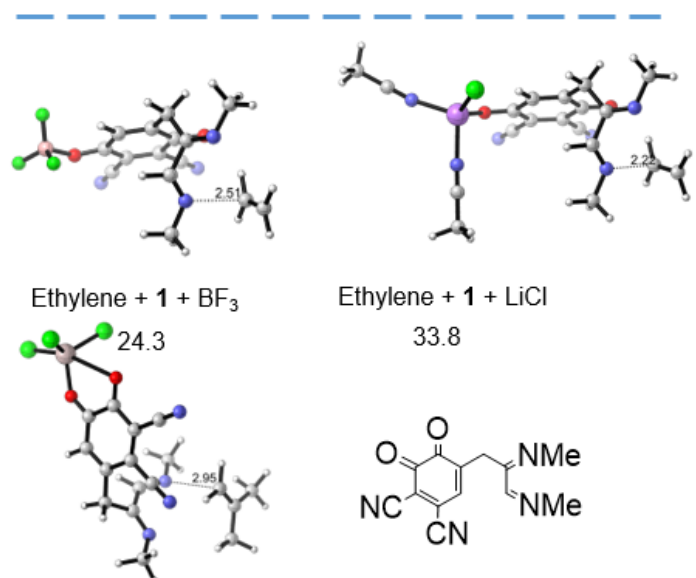

Ethylene + 1+ LiCl

Ethylene + $4+\mathrm{AlCl}_{3}$ 33.8

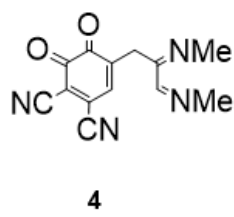

21.2

Figure 5. The geometry and relative free energy ( $\mathrm{kcal} / \mathrm{mol})$ for the $\mathbf{C P 1}\left(\mathrm{CSS}_{-}\right.$Triplet)s of selected substituted alkenes, or in the presence of Lewis acids. The key bond lengths are shown in angstrom.

\section{Summary}

In this work, we studied the formal Diels-Alder reaction between a diimine $\mathbf{1}$ with alkenes, with an intramolecular benzoquinone based oxidant. The reaction was found to proceed through a stepwise mechanism with open-shelled intermediates, either OSS or triplet, to give a biradical as the product. For most of the systems covered in this work, the first of the two $\mathrm{N}-\mathrm{C}$ bond formation events proceeds through a crossing point rather than a transition state. Once the substrate goes through the crossing point CP1, it will hop into the OSS or triplet-PES, and directly fall into the intermediate 2 with $\mathrm{N}-\mathrm{C}$ bond formed. This process is an ACET reaction, in which the ET and A events occurs in one elementary step. 
(a)

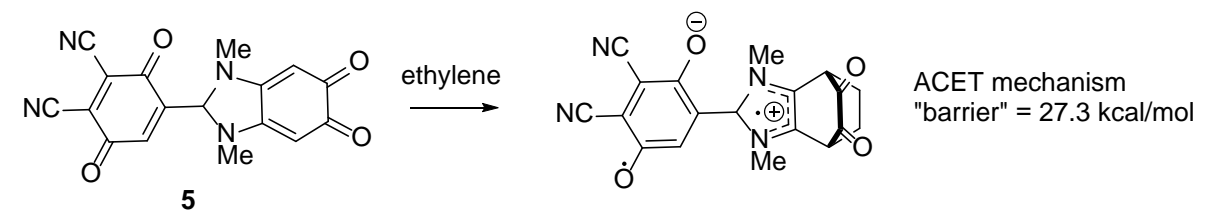

(b)

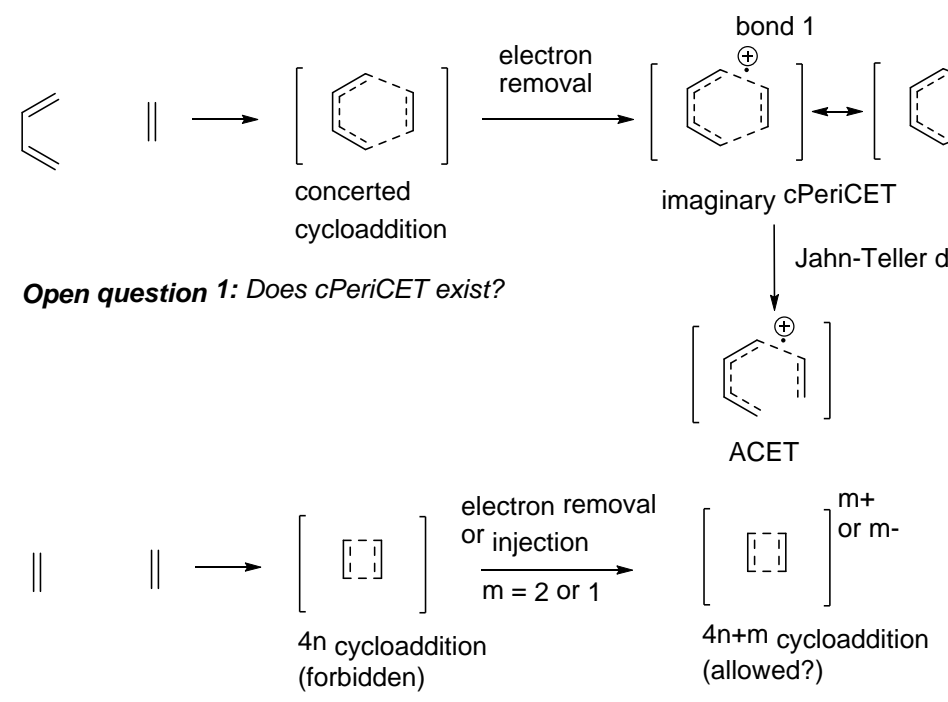

Open question 2: If cPeriCET exists, is it able to alter the electron counting rule?

Figure 6. (a) A schematic representation of the reaction of 5 with ethylene. (b) An outlook of the field of cPeriCET.21

Although the diimine $\mathbf{1}$ was taken as the model diene substrate, it is shown that the carbon-based diene substrate 5 also follows an ACET mechanism for the formal cycloaddition into ethylene, with a "barrier" determined by $\mathbf{C P 1}$ of $27.3 \mathrm{kcal} / \mathrm{mol}$ (see Supporting Information for the full PES), which is much lower than the barrier of $48.7 \mathrm{kcal} / \mathrm{mol}$ for the conventional concerted D-A. This observation indicates that the ACET-based formal cycloaddition might be not limited to some special cases.

In addition to the ACET coupled with a single electron transfer as studied above, it is of interest whether two-electron transfer in one elementary step can be coupled with a formal pericyclic reaction. Another question is whether the truly concerted Pericyclic-Coupled Electron Transfer (CPeriCET) exists. The relationship between cPeriCET and ACET is shown in Figure 6. Consider a concerted transition state which is injected or removed by one electron: the singly occupied orbital might locate in either bond 1 and bond 2. In this case, Jahn-Teller distortion might happen, shifting the mechanism into a sequence of ACETs and radical additions. In addition, if cPeriCET exists, it will be of interest if cPeriCET mechanism could alter the well-known allowing and forbidding rule of cycloadditions. As a result, it might be a continuous task to find examples for the cPeriCET mechanism, in addition to the ACET mechanism reported herein.

\section{Methods}

The Gaussian 16 package ${ }^{22}$ was employed to perform all the calculations, with the Gaussian 09 default integral grid. The wB97x-D functional ${ }^{23}$ was used for all calculations. For geometry optimization, the def2-SVP ${ }^{24}$ basis set was employed. Frequency calculations were followed to 
ensure stationary points were found, and to obtain Gibbs free energy correction at room temperature. Single point calculations were performed with the ma-def2-TZVP basis set ${ }^{25}$. Both geometry optimization and single point calculation were performed under SMD implicit solvation ${ }^{26}$ of acetonitrile. The stability of wave-function was checked for all the structures. Notably, for many of the structures, both the CSS and OSS wavefunctions are stable, although the OSS state is lower in energy, and sometimes the OSS state is rather hard to be found. The key point to reproduce the results in this work is to try various methods for the generation of initial SCF guess; the most reliable method to converge to an OSS state is to read the converged OSS wavefunction of $\mathbf{2}$ as the initial guess.

The crossing point between different PESs were located using the program KST48 written by the author ${ }^{27}$. Due to both the CSS and OSS states are stable, the location of OSS and CSS state by DFT method is relatively easy: by making use of the "read" mode in KST48, and reading the converged CSS and OSS wavefunctions for the two states, respectively, and then CP1(CSS_OSS) can be located.

The multi-reference calculations were performed by the ORCA program ${ }^{28-29}$ with the natural UHF orbital from the triplet state as the reference orbitals. In order to better compare with the DFT results under solvation, the SMD solvation model was also employed for the UHF calculation.

The spin density analysis was performed with the Multiwfn program ${ }^{30}$. The molecular geometry and isosurface were ploted with CYLView ${ }^{31}$ and $\mathrm{VMD}^{32}$.

The quasi-classical trajectory molecular dynamics simulations were performed using the PROGDYN program ${ }^{33}$. The initial geometry for each trajectory was generated by adding displacements that follows a QM-like Gaussian distribution to all vibrational modes higher than $10 \mathrm{~cm}^{-1}$ of the initial geometry. Each real normal mode was given its zero-point energy plus a random Boltzmann sampling of the thermal energy available at 298.15 K. Trajectories were propagated at wB97x-D/def2-SVP/SMD(Acetonitrile) level in both the forward and backward directions, until the product formed or the length of trajectory is longer than 200 fs. For each point the three states (CSS, OSS, Triplet) were calculated and the one with the lowest energy was used for further propagation.

\section{Acknowledgement}

The author thanks Hangzhou Yanqu Information Technology Co., Ltd. For purchasing the license of Gaussian. Also thanks to all the students in Department of Chemistry, Tsinghua University for their great love and encouragement to the author.

\section{References:}

1. Fleming, I., Pericyclic reactions. Oxford University Press, USA: 2015.

2. Ajaz, A.; Bradley, A. Z.; Burrell, R. C.; Li, W. H. H.; Daoust, K. J.; Bovee, L. B.; DiRico, K. J.; Johnson, R. P., Concerted vs Stepwise Mechanisms in Dehydro-Diels-Alder Reactions. The Journal of Organic Chemistry 2011, 76 (22), 9320-9328.

3. Beno, B. R.; Houk, K. N.; Singleton, D. A., Synchronous or Asynchronous? An "Experimental” Transition State from a Direct Comparison of Experimental and Theoretical Kinetic Isotope Effects for a Diels-Alder Reaction. Journal of the American Chemical Society 1996, 118 (41), 9984-9985.

4. Borden, W. T.; Loncharich, R. J.; Houk, K. N., Synchronicity in Multibond Reactions. Annual Review of Physical Chemistry 1988, 39 (1), 213-236. 
5. Goh, Y. W.; White, J. M., Structures of oxygenated cyclohexa-1,3-diene-maleic anyhydride cycloadducts. Structural evidence suggests a stepwise retro-Diels-Alder reaction. Organic \& Biomolecular Chemistry 2007, 5 (15), 2354-2356.

6. Houk, K. N.; Gonzalez, J.; Li, Y., Pericyclic Reaction Transition States: Passions and Punctilios, 1935-1995. Accounts of Chemical Research 1995, 28 (2), 81-90.

7. Houk, K. N.; Li, Y.; Storer, J.; Raimondi, L.; Beno, B., Concerted and stepwise mechanisms in cycloaddition reactions: potential surfaces and isotope effects. Journal of the Chemical Society, Faraday Transactions 1994, 90 (12), 1599-1604.

8. Singleton, D. A.; Schulmeier, B. E.; Hang, C.; Thomas, A. A.; Leung, S.-W.; Merrigan, S. R., Isotope effects and the distinction between synchronous, asynchronous, and stepwise Diels-Alder reactions. Tetrahedron 2001, 57 (24), 5149-5160.

9. Gonzalez-James, O. M.; Kwan, E. E.; Singleton, D. A., Entropic intermediates and hidden ratelimiting steps in seemingly concerted cycloadditions. Observation, prediction, and origin of an isotope effect on recrossing. Journal of the American Chemical Society 2012, 134 (4), 1914-1917.

10. Yang, Z.; Jamieson, C. S.; Xue, X.-S.; Garcia-Borràs, M.; Benton, T.; Dong, X.; Liu, F.; Houk, K. N., Mechanisms and Dynamics of Reactions Involving Entropic Intermediates. Trends in Chemistry 2019, 1 (1), 22-34.

11. Bai, H.-W.; Cai, Z.-J.; Wang, S.-Y.; Ji, S.-J., Aerobic Oxidative Cycloaddition of $\alpha$ Chlorotosylhydrazones with Arylamines: General Chemoselective Construction of 1,4-Disubstituted and 1,5-Disubstituted 1,2,3-Triazoles under Metal-Free and Azide-Free Conditions. Organic Letters 2015, 17 (12), 2898-2901.

12. Baker, J. R.; Hermann, A.; Wing, R. M., Mechanism of oxidative cycloaddition of olefins to metal dithiolenes. Journal of the American Chemical Society 1971, 93 (24), 6486-6489.

13. Li, Y.; Thiemann, T.; Sawada, T.; Mataka, S.; Tashiro, M., Lewis Acid Catalysis in the Oxidative Cycloaddition of Thiophenes1. The Journal of Organic Chemistry 1997, 62 (23), 7926-7936.

14. Rok Lee, Y.; Kim, B. S.; Il Kweon, H., Efficient Synthesis of Dihydrofuroquinolinones and Furoquinolinones by Silver(I)/Celite Promoted Oxidative Cycloaddition. Tetrahedron 2000, 56 (24), 3867-3874.

15. Zhang, Y.; Li, X.; Li, J.; Chen, J.; Meng, X.; Zhao, M.; Chen, B., CuO-Promoted Construction of N-2-Aryl-Substituted-1,2,3-Triazoles via Azide-Chalcone Oxidative Cycloaddition and Post-Triazole Arylation. Organic Letters 2012, 14 (1), 26-29.

16. Cañellas, S.; Montgomery, J.; Pericàs, M. À., Nickel-Catalyzed Reductive [2+2] Cycloaddition of Alkynes. Journal of the American Chemical Society 2018, 140 (50), 17349-17355.

17. Jenkins, A. D.; Herath, A.; Song, M.; Montgomery, J., Synthesis of Cyclopentenols and Cyclopentenones via Nickel-Catalyzed Reductive Cycloaddition. Journal of the American Chemical Society 2011, 133 (36), 14460-14466.

18. Zhou, Y.-Y.; Uyeda, C., Catalytic reductive [4 +1$]$-cycloadditions of vinylidenes and dienes. Science 2019, 363 (6429), 857-862.

19. Chang, H.-T.; Jayanth, T. T.; Cheng, C.-H., Cobalt-Catalyzed Diastereoselective Reductive [3 + 2] Cycloaddition of Allenes and Enones. Journal of the American Chemical Society 2007, 129 (14), 41664167.

20. Ma, Y., Addition Coupled Electron Transfer (ACET) and Addition Coupled Electron Coupled Proton Transfer (ACPCET). 2021.

21. Angeli, C.; Cimiraglia, R.; Evangelisti, S.; Leininger, T.; Malrieu, J.-P., Introduction of n-electron 
valence states for multireference perturbation theory. The Journal of Chemical Physics 2001, 114 (23), 10252-10264.

22. Frisch, M. J.; Trucks, G. W.; Schlegel, H. B.; Scuseria, G. E.; Robb, M. A.; Cheeseman, J. R.; Scalmani, G.; Barone, V.; Petersson, G. A.; Nakatsuji, H.; Li, X.; Caricato, M.; Marenich, A. V.; Bloino, J.; Janesko, B. G.; Gomperts, R.; Mennucci, B.; Hratchian, H. P.; Ortiz, J. V.; Izmaylov, A. F.; Sonnenberg, J. L.; Williams; Ding, F.; Lipparini, F.; Egidi, F.; Goings, J.; Peng, B.; Petrone, A.; Henderson, T.; Ranasinghe, D.; Zakrzewski, V. G.; Gao, J.; Rega, N.; Zheng, G.; Liang, W.; Hada, M.; Ehara, M.; Toyota, K.; Fukuda, R.; Hasegawa, J.; Ishida, M.; Nakajima, T.; Honda, Y.; Kitao, O.; Nakai, H.; Vreven, T.; Throssell, K.; Montgomery Jr., J. A.; Peralta, J. E.; Ogliaro, F.; Bearpark, M. J.; Heyd, J. J.; Brothers, E. N.; Kudin, K. N.; Staroverov, V. N.; Keith, T. A.; Kobayashi, R.; Normand, J.; Raghavachari, K.; Rendell, A. P.; Burant, J. C.; Iyengar, S. S.; Tomasi, J.; Cossi, M.; Millam, J. M.; Klene, M.; Adamo, C.; Cammi, R.; Ochterski, J. W.; Martin, R. L.; Morokuma, K.; Farkas, O.; Foresman, J. B.; Fox, D. J. Gaussian 16 Rev. C.01, Wallingford, CT, 2016.

23. Chai, J.-D.; Head-Gordon, M., Long-range corrected hybrid density functionals with damped atomatom dispersion corrections. Physical Chemistry Chemical Physics 2008, 10 (44), 6615-6620.

24. Weigend, F.; Ahlrichs, R., Balanced basis sets of split valence, triple zeta valence and quadruple zeta valence quality for H to Rn: Design and assessment of accuracy. Physical Chemistry Chemical Physics 2005, 7 (18), 3297-3305.

25. Zheng, J.; Xu, X.; Truhlar, D. G., Minimally augmented Karlsruhe basis sets. Theoretical Chemistry Accounts 2011, 128 (3), 295-305.

26. Marenich, A. V.; Cramer, C. J.; Truhlar, D. G., Universal solvation model based on solute electron density and on a continuum model of the solvent defined by the bulk dielectric constant and atomic surface tensions. The Journal of Physical Chemistry B 2009, 113 (18), 6378-6396.

27. Ma, Y. KST48: A Powerful Tool for MECP location. https://github.com/RimoAccelerator/KST48 (accessed February 1).

28. Neese, F.; Wennmohs, F.; Becker, U.; Riplinger, C., The ORCA quantum chemistry program package. The Journal of Chemical Physics 2020, 152 (22), 224108.

29. Neese, F., Software update: the ORCA program system, version 4.0. Wiley Interdisciplinary Reviews: Computational Molecular Science 2018, 8 (1), e1327.

30. Lu, T.; Chen, F., Multiwfn: a multifunctional wavefunction analyzer. Journal of computational chemistry 2012, 33 (5), 580-592.

31. Legault, C., CYLview, 1.0 b. Université de Sherbrooke 2009, 436, 437.

32. Humphrey, W.; Dalke, A.; Schulten, K., VMD: visual molecular dynamics. Journal of molecular graphics 1996, 14 (1), 33-38.

33. Singleton, D. A.; Hang, C.; Szymanski, M. J.; Greenwald, E. E., A New Form of Kinetic Isotope Effect. Dynamic Effects on Isotopic Selectivity and Regioselectivity. Journal of the American Chemical Society 2003, 125 (5), 1176-1177. 